\title{
Ações/plano de alta da enfermagem à mulher submetida à mastectomia
}

RESUMO | Objetivo: analisar produções científicas de enfermagem dos últimos dez anos que abordem as ações e orientações para o plano de alta de mulheres submetidas à mastectomia. Método: Para a realização da busca de artigos realizou uma revisão sistemática de artigos utilizados - estratégia PICO, nos idiomas Português, Inglês e Espanhol respectivamente. Resultados: Foram utilizadas 19 publicações para a posterior discussão, onde 15 eram de síntese qualitativa e 4 de síntese quantitativa. Conclusão: A maioria dos artigos evidenciou que as intervenções de enfermagem no plano de alta a mulheres submetidas à mastectomia foram baseadas na educação continuada dessas mulheres e também de seus familiares. Essa ferramenta consiste em orientações baseadas na utilização de drenos, apoio emocional, possibilidade de esvaziamento axilar, identificação de sinais de infecções e manejo nos curativos.

Palavras-chaves: Alta do Paciente; Cuidados de Enfermagem; Mastectomia.

ABSTRACT | Objective: to analyze scientific nursing productions from the last ten years that address the actions and guidelines for the discharge plan of women undergoing mastectomy. Method: To carry out the search for articles, a systematic review of articles used was carried out - PICO strategy, in Portuguese, English and Spanish respectively. Results: 19 publications were used for further discussion, where 15 were of qualitative synthesis and 4 of quantitative synthesis. Conclusion: Most articles showed that nursing interventions in the discharge plan for women undergoing mastectomy were based on the continuing education of these women and their families. This tool consists of guidelines based on the use of drains, emotional support, the possibility of axillary emptying, identification of signs of infections and management of dressings.

Keywords: Patient Discharge; Nursing Care; Mastectomy.

RESUMEN | Objetivo: analizar las producciones científicas de enfermería de los últimos diez años que abordan las acciones y pautas para el plan de alta de las mujeres sometidas a mastectomía. Método: para llevar a cabo la búsqueda de artículos, se realizó una revisión sistemática de los artículos utilizados: estrategia PICO, en portugués, inglés y español, respectivamente. Resultados: se utilizaron 19 publicaciones para una discusión adicional, donde 15 fueron de síntesis cualitativa y 4 de síntesis cuantitativa. Conclusión: La mayoría de los artículos mostraron que las intervenciones de enfermería en el plan de alta para mujeres sometidas a mastectomía se basaron en la educación continua de estas mujeres y sus familias. Esta herramienta consta de pautas basadas en el uso de drenajes, apoyo emocional, posibilidad de vaciado axilar, identificación de signos de infecciones y manejo de apósitos.

Descriptores: Alta del Paciente; Cuidados de Enfermería; Mastectomía.

\section{Bruna dos Santos Scofano}

Mestre, pelo Programa Acadêmico em Ciências do Cuidado em Saúde (PACCS), na Universidade Federal Fluminense (UFF) e enfermeira especialista em oncologia, pela Universidade Estadual do Rio de Janeiro (UERJ), RJ, Brasil.

\section{Andreia Almeida de Lima}

Enfermeira especialista em oncologia, pela Universidade Estadual do Rio de Janeiro (UERJ) e especialista em enfermagem clínica pela UERJ, RJ, Brasil.

\section{Rhaiza dos Reis Silva}

Enfermeira especialista em oncologia, pela UERJ e especialista nos moldes de residência: clínica e cirurgia geral pela Universidade Federal do Estado do Rio de Janeiro (UNIRIO), RJ, Brasil.

Recebido em: 03/02/2020

Aprovado em: 04/02/2020

\section{Lucia Helena Garcia Penna}

Dr Professora Adjunto do Departamento de Enfermagem Materno Infantil e do Programa de Pós-Graduação em Enfermagem da UERJ.

\section{Karla Biancha Silva de Andrade}

Enfermeira Gestora da Qualidade da Unidade de Emergência do INCA; Professora Adjunta do Departamento de Enfermagem Médico-Cirúrgico, Docente responsável pelo programa teórico da Residência em Enfermagem Cardiovascular e Coordenadora do curso de pós graduação lato senso Enfermagem em Oncologia e enfermeira Coordenadora do Centro de Tratamento Intensivo do Instituto Nacional de Câncer José Alencar Gomes da Silva (INCA) - Unidade II.

\section{Ana Paula Brito Pinheiro}

Professora Adjunta da Coordenação da Pós Graduação de Oncologia em Enfermagem da UERJ e Doutoranda em Enfermagem e Biociências pela UNIRIO.
INTRODUÇÃo

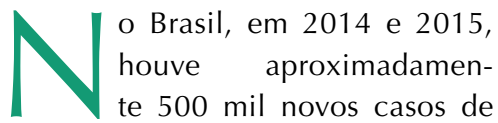
câncer, o que reforça a discussão sobre o problema no país, e seu controle e prevenção como prioridades em todos os estados ${ }^{(1)}$.

Além disso, todos os anos verifica-se que um milhão de mulheres são diagnosticadas com câncer de mama em todo o mundo e, infelizmente, estima-se que 420.000 morrerão dessa doença. O câncer de mama é mais elevado em países desenvolvidos, sendo que as maiores incidências ocorrem no Reino Unido, Austrália, Estados Unidos da América (EUA) e Canadá. Embora a mortalidade de pacientes com este tipo de doença ainda esteja elevada em vários países, os EUA, Reino Unido e Austrália já registram diminuição da 
mortalidade, atribuída ao uso ampliado de mamografia e ao tratamento precoce da doença $\mathrm{a}^{(2)}$.

Em mulheres jovens, o câncer de mama ocorre em 5 a $7 \%$ dos casos. Ressalta-se que antes dos 50 anos, esse tipo de doença apresenta-se com pior prognóstico, uma vez que, na maioria das vezes, o diagnóstico é feito somente a quando a paciente é sintomática e, portanto, já evoluiu para um estágio mais avançado da doença ${ }^{(3)}$.

No que diz respeito aos dados estatísticos sobre o câncer de mama em homens, esta doença representou menos de $1 \%$ de todos os casos e $0,1 \%$ da mortalidade por câncer no sexo masculino em 2015. Apesar desses dados, alguns estudos indicam que a incidência de homens submetidos à mastectomia vem aumentando de 0,86 para 1,08 por $100.000^{(4)}$.

Nesse contexto, o plano terapêutico multiprofissional para os pacientes com câncer de mama deve ser elaborado após a confirmação do diagnóstico e conforme os fatores associados ao tumor e ao próprio paciente. Em determinadas situações, é levado em conta o perfil gênico. No caso de pacientes femininas, a decisão terapêutica é tomada depois da verificação de elementos primordiais, como por exemplo: o estado menopausa, a idade, existência de uma ou mais doenças e níveis de entendimento socioculturais acerca da decisão do tratamento ${ }^{(5)}$.

Ainda, as principais condutas usadas pelo enfermeiro ao colocar em prática os cuidados na recuperação da mulher submetida à mastectomia, estão embasadas na prevenção de complicações relacionadas à incisão cirúrgica ao dreno, à reabilitação física e também as questões associadas ao sentimento e o medo da paciente ${ }^{(5)}$.

Na promoção da saúde há uma valorização do autocuidado, considerando a participação da própria mulher submetida à mastectomia no processo de enfrentamento, prevenção de com- plicações, recuperação e reabilitação após a cirurgia ${ }^{(5)}$.

O apoio e a orientação não somente para as mulheres mastectomizadas, mas também para a família, que é a parte importante no tratamento, pois, se o suporte emocional for de qualidade, será bastante significativo na recuperação da mulher, ajudando-a na melhora da autoestima e contribuindo na recuperação da autoimagem ${ }^{(6)}$.

O enfermeiro é um profissional educador e, por ter essa formação, deve orientar a mulher e também aos seus familiares quanto ao pós-operatório da mastectomia, uma vez que esse período é o mais traumático para ambos ${ }^{(7)}$.

O cuidado do enfermeiro está baseado no amor, compaixão, carinho, não sendo visto apenas como o tratamento de uma doença, mas sim, como uma possibilidade do ser de quem é cuidado. Então, ouvir, tocar, estar disponível é uma forma de promover um tratamento humanizado além de promover o resgate e o cuidado que, na cultura científica, foi desprezado e colocado em suspeita por ser de natureza subjetiva ${ }^{(8)}$.

O vínculo estabelecido entre profissional e o cliente favorece a contribuinte do autocuidado para além do contexto da unidade de saúde, a partir de uma relação de confiança, o enfermeiro poderá estabelecer orientações para alta oferecendo e contribuindo com o cuidado.

Nesse aspecto, o conceito de Plano de Alta é caracterizado pela forma organizada de realizar determinadas ações de acordo com as condições individuais de cada paciente após a alta, devendo ser formulado com a participação de todos os profissionais de saúde que estão envolvidos de maneira direta com o paciente ${ }^{(9)}$.

O plano de alta é importante na promoção da saúde de mulheres submetidas à mastectomia. Para sua realização, o enfermeiro deve compreender a complexidade da situação de cada mulher, elaborar o plano com a colabo- ração dos outros profissionais de saúde, visando o atendimento integral de suas necessidades afim de oferecer um meIhor prognóstico para essa mulher.

A relevância de se conhecer o que vem sendo abordado sobre essas ações/ orientações, justifico este estudo, o qual tem por questão principal: Quais são as ações utilizadas pelo enfermeiro na realização do plano de alta hospitalar a mulheres mastectomizadas?

Considerando a inquietação e a escassez de produções específicas atuais sobre as orientações/ações às mulheres submetidas à mastectomia, buscamos com esse estudo analisar produções científicas de enfermagem dos últimos cinco anos que abordem as ações e orientações para o plano de alta de muIheres submetidas à mastectomia.

\section{METODOLOGIA}

Trata-se de um estudo de revisão integrativa que é fundamentada na coleta de dados disponíveis na literatura para compará-los e aprofundar o conhecimento do tema investigado ${ }^{(10)}$.

Esta pesquisa tem com o objetivo de identificar, analisar e discutir as ações do enfermeiro na realização do planejamento de alta hospitalar em mulheres mastectomizadas.

Em que os critérios de inclusão são: artigos em Português, Inglês e Espanhol; recorte temporal dos últimos cinco anos (de 2013 a maio de 2018) e publicações com foco nas ações do enfermeiro no planejamento de alta hospitalar a pacientes mastectomizadas.

A estratégia de pesquisa deu-se da seguinte maneira: foi realizada em Maio de 2018. No PubMed via MEDLINE, ((Nursing Care) AND Patient Discharge) OR Mastectomy; Cochrane Library, ("Nursing Care" AND "Patient Discharge" OR "Mastectomy"); Scopus, ("Nursing Care" AND "Patient Discharge" OR "Mastectomy"), ("Nursing" AND "Mastectomy"), ("Nursing" AND "Patient Discharge"), ("Nursing" AND "Patient 
Discharge"); CINAHL, ("Nursing Care" AND "Patient Discharge" OR "Mastectomy") e LILACS via BVS, (tw:(Cuidados em Enfermagem)) AND (tw:(Alta do Paciente)) OR (tw:(Mastectomia)).

Foi utilizado o software ZOTERO

$\begin{aligned} & \text { Quadro 1. Estratégia PICO, nos idiomas Português, Inglês e Espanhol respectivamente. } \\
& \text { Rio de Janeiro, RJ, Brasil, } 2019\end{aligned}$
\begin{tabular}{l|l|} 
P (paciente/população) & $\begin{array}{l}\text { Publicações de Enfermeiros (Nursing Publica- } \\
\text { tions/ Publicaciones de enfermeros) }\end{array}$ \\
\hline I (intervenção/ fenômeno de interesse) & $\begin{array}{l}\text { Plano de alta hospitalar (Discharge plan/ } \\
\text { Plan de alta hospitalaria) }\end{array}$ \\
\hline C o (controle ou comparação/ contexto) & $\begin{array}{l}\text { Mulher mastectomizada (Mastectomized } \\
\text { woman / Mujer mastectomizada) }\end{array}$ \\
\hline
\end{tabular}

Fonte: Adaptado de Santos, Pimenta e Nobre ${ }^{(11)}$.

\section{Figura 1. Diagrama PRISMA para o processo de triagem dos estudos. Rio de Janeiro, RJ, Brasil, 2019}



versão 5.0 para gerenciar as publicações encontradas. A análise dos dados foi elaborada por meio do checklist Principais Itens para Relatar Revisões sistemáticas e Meta-análises - PRISMA(12), em que foi elaborado um diagrama (Figura 1) para a extração dos dados, que foi revisado pelas pesquisadoras.

\section{RESULTADOS}

Ao realizar o somatório de todos os artigos que atenderam aos critérios de inclusão pré-estabelecidos, totalizou-se 1.025 publicações, onde 1.010 eram registros identificados através de bancos de dados e 15 eram outros registros identificados através de outras fontes.

Neste contexto, com a aplicabilidade dos critérios de exclusão: das 1.025 publicações analisadas pela categoria texto duplicado, restaram apenas 990. Das 990 publicações que passaram pela etapa de rastreamento, restaram apenas 32. Das 32 publicações rastreadas, 7 foram excluídas, restando somente 25. Das 25 publicações avaliadas pela categoria texto completo, nenhuma foi excluída. Foram utilizadas 25 publicações para a posterior discussão, onde 15 eram de síntese qualitativa e 10 de síntese quantitativa.

Fonte: Adaptado de Santos, Pimenta e Nobre ${ }^{(11)}$.

Quadro 2. Descrição dos estudos. Rio de Janeiro, RJ, Brasil, 2019.

\section{Título/ Autor}

As experiências das enfermeiras da enfermaria no processo de alta entre a unidade de terapia intensiva e a enfermaria geral.

KAUPPI, W.; PROOS, M. e Olausson, S. 2018.

Qualidade de vida em mulheres adultas mastectomizadas.

RODRIGUES, Clara, 2017.

Construção de instrumentos para o cuidado sistematizado da enfermagem: Mulheres em processo cirúrgico de mastectomia

PAIVA, A.C.P.C, et al 2016.
Ações do enfermeiro no plano de alta hospitalar a mulheres mastectomizadas

Intervenção: comunicação com a família do paciente com o objetivo de realizar orientações após a alta.

Orientações sobre os drenos e dos pontos da ferida cirúrgica, esclarecimentos sobre dor e o medo, mobilização articular, drenagem linfática manual.

Os diagnósticos, as intervenções e os resultados de enfermagem foram registrados segundo NANDA International, Inc, Nursing Intervention Classification e Nursing Outcome Classification respectivamente, sendo selecionados 25 possíveis diagnósticos, 39 intervenções vinculadas ao processo cirúrgico e 42 resultados para atender às necessidades de informação, enfrentamento e apoio. Estabeleceram-se parâmetros para mensurar o impacto do cuidado. 
Qualidade de vida entre os pacientes após mastectomia profilática bilateral: uma revisão sistemática dos resultados relatados pelo paciente.

RAZDAN, S.N, et al 2016.

Resultados para os cuidadores familiares de uma intervenção de alta hospitalar prestada por enfermeiros para pessoas idosas (o Programa Habilitação em Casa): Ensaio controlado aleatorizado simples cego

TOYE, C, et al. 2016.

Cuidados sensíveis de enfermagem a mulheres submetidas à mastectomia: subsídios para uma ação educativa com enfoque na dimensão ética e estética

NICOLAU, S R T C, 2015.

Efeito de um programa educacional de planejamento de alta em um hospital universitário

SAKAI, S., et al. 2015.

Atuação do enfermeiro no preparo para a alta hospitalar de pacientes cirúrgicos

MARTINS, K.P, et al.

2015.

Planejamento da alta: melhores práticas em transições de cuidados

PELLETT, C. 2014.

Opinião das mulheres sobre a alta hospitalar de 23 horas após mastectomia

LAMBERT, L e RUSBY, J 2014.

0 planejamento da alta se volta para a beira do leito: uma abordagem centrada no paciente e na família

WROBLESKI, D.M., et al 2014.

Protocolo clínico para o cuidado de enfermagem a mulheres mastectomizadas: um estudo exploratório descritivo

NICOLAU, S. R. T. C; TEIXEIRA, E. R 2014.

Práticas de alta para o paciente em terapia intensiva: uma exploração qualitativa no ambiente da enfermaria geral

COGNET, S; COYER, F. 2014.

Mastectomia por câncer de mama no Brasil: distribuição geográfica e custos na perspectiva da saúde pública

VALLE, P.M., et al 2014.

Alta hospitalar: resultados de um estudo prospectivo multicêntrico italiano usando Blaylock Risk Assessment Screening Score.

DAL MOLIN, A., et al. 2014
A intervenção utilizada para o estudo foi a implementação do termo qualidade de vida relacionado à saúde em mulheres que realizaram a mastectomia bilateral profilática .

Intervenções: Obter ajuda, informações e recursos; desenvolver planos de crise e obter referências de serviços e organizar requisitos legais.

Orientações verbais, sobre o dreno, autocuidado, não há sistematização.

Intervenção: programa educacional de planejamento de alta na equipe multidisciplinar.

Identificou-se que as principais estratégias foram as orientações referentes aos cuidados básicos aos familiares. Além disso, também foi identificado que o enfermeiro não insere o familiar durante a assistência, dificultando a continuidade do cuidado no domicílio.

A intervenção: avaliação do paciente, encaminhamento as outras instituições e fornecimento de endereços para serviços comunitários e equipes hospitalares.

0 estudo fornece evidências para apoiar a visão de que a alta de 23 horas é viável do ponto de vista médico e aceitável para os pacientes.

Intervenção: foram realizadas orientações após a alta do paciente.

Considera-se o desenvolvimento do Protocolo proposto neste trabaIho um recurso essencial para guiar o enfermeiro no planejamento da assistência, desde a fase ambulatorial até a internação e a alta hospitalar da paciente mastectomizada. Além de ser um instrumento para a educação do paciente, visando ao seu autocuidado e à adoção de comportamentos adequados para que a paciente tenha uma maior qualidade de vida após a mastectomizada

Intervenção: comunicação com a família do paciente com o objetivo de realizar orientações após a alta

Intervenção: comunicação com a família do paciente com o objetivo de realizar orientações após a alta

Os resultados indicam que o índice BRASS é útil para identificar pacientes com risco de hospitalização prolongada. 
Processo de alta: um projeto para melhorar a qualidade e a pontualidade das prescrições completas.

KANNAKERIL, G., et al 2014.

Intervenção do enfermeiro no tratamento quimioterápico de mulheres com câncer de mama SOARES, S.G.SC; ALBUQUERQUE, J.O.L, 2014.

Planejamento de alta e avaliação de riscos de enfermeiros: comportamentos, compreensão e barreiras.

GRAHAM, J., et al 2013.

Eficácia da informação de alta da assistência crítica no apoio à recuperação precoce da doença crítica

BENCH, S., et al 2013.

Considerações sobre os cuidados de enfermagem aos pacientes na alta hospitalar: revisão integrativa

SOUZA, M. B. B; CARVALHO, P. e QUELUCI, C. G, 2013.

Educar como prática facilitadora do processo de cuidar às mulheres mastectomizadas: revisão integrativa

BORDALLO, F.R et al 2013

Um ensaio randomizado de intervenções de enfermagem que apoiam a recuperação do paciente pós-mastectomia.

CHO, H.S.M., et al 2013

Promoção do autocuidado a mulheres mastectomizadas.

CARVALHO, A.P.R; SANTOS, T.M.B e LINHARES, F.M.P, 2012

Qualidade de vida e cuidado de enfermagem na percepção de mulheres mastectomizadas

ALMEIDA, N.G et al, 2015

0 cuidado de enfermagem vivenciado por mulheres mastectomizadas.

SILVA, G.N.C et al, 2013.
Intervenção: medicação eletrônica e guia on line

Apoio emocional e encaminhamento para equipe multidisciplinar, quando apresenta sinais de depressão pós mastectomia. Fornece informações sobre a busca de soluções dos problemas relacionados ao tratamento; instrumentalizá-las para que tomem decisões sobre o tratamento proposto; e levar ao desempenho de ações de autocuidado.

enfermeiros requerem incentivo e apoio adicionais para cumprir as políticas de planejamento de alta, e as políticas de planejamento de alta devem ser adaptadas para lidar melhor com trajetórias imprevisíveis de doenças.

São utilizadas informações individualizadas após a alta da paciente mastectomizada.

Intervenção: a comunicação multidisciplinar e a ênfase na situação-problema do paciente no processo de alta.

As ações educativas para as mulheres mastectomizadas foram representadas por grupos de apoio e manuais educativos.

São utilizadas informações individualizadas após a alta da paciente mastectomizada

Não fazer esforço com o braço do lado da mastectomia, não posso tomar injeção, não expor a temperatura quente, não verificar a pressão arterial. Cuidados com o curativo, o dreno e a continuação do tratamento após a cirurgia, massagem linfática.

Orientações sobre a troca de dreno, higiene, apoio emocional, ensino aprendizagem, medicamentos.

Orientações sobre medicamentos, autocuidado, não fazer esforço físico com o braço do lado da mastectomia.

\section{DISCUSSÃO}

As ações e orientações para o plano de alta de mulheres submetidas à mastectomia

Em um estudo ${ }^{(14)}$ realizado para identificar as principais ações de enfermagem no plano de alta a mulheres mastectomizadas apontou que há 39 intervenções de enfermagem e 42 resultados relacionados a mastectomia baseados na abordagem integral, humanizada e individualizada. Além disso, essas intervenções consideram as necessidades reais dessas mulheres a partir dos problemas apresentados por elas, como por exemplo: medo, queixas, dúvidas, anseios e intercorrências sobre a mastectomia.

Dessa forma, pode-se dizer que a abordagem humanizada à mulher mastectomizada e aos seus familiares é com- posta por ações que originem espaços que permitam a todos expressar seus sentimentos e também a valorizá-los; identificar áreas problemáticas; ajudá- los a identificar fontes de ajuda, que podem estar dentro ou fora da própria família; fornecer orientações e elucidar suas percepções; ajudá-los a solucionar os problemas associados ao tratamento da mastectomia; instrumentalizá-los para 
que tomem decisões sobre o tratamento proposto; e levar ao desempenho de ações de autocuidado, de acordo com as suas possibilidades ${ }^{(15)}$.

Nesse aspecto, complementa-se que a comunicação é necessária durante este processo e deve ser eficaz, seja ela verbal, por palavras, ou não-verbal, pelos gestos e expressões. O enfermeiro precisa conversar, comunicar orientar e esclarecer a paciente sobre o seu tratamento, os procedimentos que serão realizados e o seu atual quadro clínico ${ }^{(16)}$.

Em contrapartida, em um outro estudo $^{(17)}$ sobre as intervenções de enfermagem em 26 participantes do sexo feminino que foram submetidas à mastectomia identificou que a melhor abordagem é receber orientações individualizadas estabelecidas no plano de alta antes do horário previsto para a saída formal da mulher do hospital, evitando o acúmulo de informações nesse momento, assegurando a avaliação de uma melhor compreensão, com a finalidade de esclarecer dúvidas. Nesse aspecto, o enfermeiro, no momento da alta, deve reforçar as orientações sobre o plano a ser seguido e a importância do retorno da mulher para o controle e a reabilitação de sua saúde, procurando a equipe sempre que necessitar de auxílio.

Dessa forma, um dos maiores problemas que levam as mulheres com mastectomia a procurarem a equipe, em especial o enfermeiro, era a depressão após o procedimento e a ocorrência de infecções no local da incisão. Nesse caso, é necessário que este profissional tenha um olhar integral com essas mulheres a fim de aumentar a qualidade de suas orientações pós-alta ${ }^{(18)}$.

Contudo, em um estudo controlado randomizado $^{(19)}$, simples-cego realizado sobre a comparação entre dois grupos onde um utilizava um programa ministrado por uma enfermeira especialmente treinada por telefone que desempenhava as seguintes atividades no plano pós alta: apoio para facilitar a compreensão do plano de alta da paciente mastectomiza- da; avaliação das necessidades de apoio do cuidador; priorização do cuidador de necessidades urgentes; e orientação colaborativa, do enfermeiro, quanto ao acesso aos suportes e o outro não utilizava nenhum plano de cuidado, apontou que o grupo que recebeu a intervenção apresentou melhoras no que diz respeito ao processo cirúrgico.

Ainda, acrescenta-se que há diversas ferramentas que facilitam a parceria da paciente submetida à mastectomia e à família. Uma dessas ações é a conferência de planejamento de alta, na qual os enfermeiros, a paciente e, em alguns casos, seus cuidadores familiares, participam de um debate sobre a realização de um plano de transição dos cuidados da paciente para outro ambiente, como por exemplo o domicílio(20).

$\mathrm{Na}$ tentativa de personalizar as orientações, demonstrou-se em um que o fornecimento de livros para o registro de perguntas e possíveis preocupações acerca da mastectomia se mostrou eficaz no planejamento pós-alta, uma vez que as pacientes mastectomizadas podem ver esta ferramenta como uma oportunidade de expressar seus sentimentos e esclarecer suas dúvidas sem a interferência de outras pessoas. Além disso, por meio desta ferramenta, o enfermeiro poderá analisar o tipo de abordagem que ele terá com a paciente ${ }^{(21)}$.

Ademais, a educação continuada com a paciente mastectomizada é uma estratégia favorável para ser inserida no planejamento pós-alta, pois garante o conforto e bem-estar da mulher após alta hospitalar. Essa ferramenta envolve orientações acerca do pós operatório, tais como: uso de drenos (recomendar o uso de roupas mais largas e esvaziar o coletor duas vezes ao dia, caso a paciente utilize dreno), curativos (recomendar a sua troca todos os dias, sempre protegendo-os com plástico para que não fiquem molhados no banho), caso a paciente tenha sido submetida ao esvaziamento axilar, recomendar que a paciente evite contato com substâncias irritantes ou cortantes com o lado operado, evite sol no período de 10 da manhã às 16 horas, e evite carregar objetos pe- sados), possibilidade de infecções (sinais de vermelhidão e inchaço no local da cirurgia ou no braço do local operado) e apoio emocional - recomendar a paciente a expressar os seus sentimentos, medos e angústias, além de esclarecer dúvidas sobre o procedimento cirúrgico ${ }^{(22)}$.

Em um estudo observacional, multicêntrico e prospectivo ${ }^{(23)}$ desenvolvido em seis hospitais italianos sobre as intervenções de enfermagem em 711 pacientes mastectomizadas evidenciou que o grupo de apoio estabelecido por enfermeiros após a alta melhora o enfrentamento para situações difíceis, tais como: o medo, anseios, sentimentos de rejeição e angústia.

Corroborando com os autores supracitados, a preocupação com ações educativas voltadas para a mulher mastectomizada, onde os saberes circulam, se constroem e se reconstroem nos discursos e nos compartilhamentos de informações, principalmente feitos em grupos de apoio. $\mathrm{O}$ autor $^{(24)}$ esclarece que o grupo de apoio após a alta, incentivado pelo enfermeiro, proporciona um ambiente que oferece o suporte social, compartilhamento de sentimentos, desenvolvimento de habilidades para enfrentamento de situações difíceis, educação em saúde, informação e debates sobre as questões existenciais da mulher submetida à mastectomia.

Em um estudo ${ }^{(25)}$ feito com nove muIheres mastectomizadas, entre quatro e oito semanas após a cirurgia, apontou que a discussão sobre os seus pontos de vista de maneira mais aberta sobre a mastectomia tornou-se algo favorável para o plano de alta. Constatou-se, também, que as complicações no pós-operatório foram bem gerenciadas e todos as participantes foram tranquilizadas por ter o número de contato do enfermeiro especialista.

Todavia, em um trabalho desenvolvido sobre as orientações (possibilidade do uso de dreno, esvaziamento axilar, curativos e esclarecimento das dúvidas sobre o procedimento a ser realizado) feito no pré-operatório em 145 mulheres submetidas à mastectomia reduziu o número de reinternações 
pós-alta, uma vez que as pacientes tinham um maior conhecimento sobre as complicações neste período ${ }^{(26)}$.

Em um estudo ${ }^{(27)}$ realizado com 256 enfermeiros em um hospital universitário nacional de 1.210 leitos na área de Tóquio acerca das intervenções de enfermagem no processo de pós alta em mulheres mastectomizadas avaliou dois grupos, um grupo de educação composto por 102 enfermeiros de 4 unidades e um grupo de controle composto por 154 enfermeiros de 6 unidades. Foram analisadas as informações obtidas de 87 enfermeiros do grupo de educação e 104 enfermeiros do grupo de controle. No grupo de educação, o conhecimento aumentou após a intervenção em relação aos serviços de enfermagem de visita domiciliar. Além disso, o grupo de educação mostrou melhora nas atitudes em relação ao planejamento de alta. Da mesma forma, o reconhecimento de necessidades de planejamento de alta por parte dos enfermeiros foi significativamente aprimorado nas unidades do grupo de educação em comparação com as unidades do grupo de controle.

Em uma intervenção para melhorar o processo e a qualidade do plano de alta em mulheres mastectomizadas deve abordar diretamente essas questões e ajudar a aumentar o conhecimento e a conscientização para que o acompanhamento seja feito corretamente. $\mathrm{O}$ autor ${ }^{(28)}$ recomenda um guia on-line desenvolvido para fornecer orientações claras e úteis aos enfermeiros e outros profissionais de saúde sobre os pacientes que receberam alta e o que eles devem orientar a essas pessoas.

Outra ação importante que pode ser desempenhada pelo enfermeiro é a elaboração de um folheto informativo sobre os cuidados a pacientes que foram submetidas à mastectomia, que será entregue às pacientes e explicado pelo enfermeiro durante as orientações para a alta hospitalar. Neste folheto, deve conter informações sobre as complicações, como: hemorragias, seromas, infecções, necrose de pele, lesão de nervo, diminuição de movimento e linfedema e outras intercorrências que possam surgir ${ }^{(29)}$.

Incluir o folheto informativo é uma conduta benéfica para a paciente, pois uniformiza as informações oferecidas quanto aos exercícios bem como cuidados a partir daquele momento. Uma adaptação física e emocional da mulher é essencial no que diz respeito à promoção da sua recuperação funcional, que se reflete diretamente na sua independência precoce para o autocuidado ${ }^{(30)}$.

Atenta-se, também, que após a avaliação da paciente mastectomizada para o desenvolvimento do autocuidado, inicia-se a sua preparação, da família ou do responsável pelo autocuidado para se tornar independente da atuação do enfermeiro. Se a paciente apresenta sistema de drenagem aspirativo, é essencial que ela e o seu familiar sejam orientados quanto à necessidade de atenção ao aspecto da drenagem; observar alterações no local do dreno (hiperemia, exsudato, dor); medir o conteúdo drenado pelo menos duas vezes ao dia, sempre lavando as mãos antes e após o procedimento; anotar a data, hora e quantidade da drenagem; manter vigilância para prevenir obstruções, quando se deve realizar a ordenha leve ${ }^{(31)}$.

É dessa forma que o enfermeiro busca mudar a atitude, onde são incorporados bases e princípios que regem os fundamentos científicos, com o objetivo de inseri-las na prática diária. O cuidado à mulher com câncer de mama precisa ser desenvolvido com a finalidade de estabelecer apoio emocional prestado pela enfermagem e equipe multiprofissional, em contribuição à diminuição de complicações cognitivas, como: percepção, atenção, memória, raciocínio e imaginação afetivas e comportamentais decorrentes do tratamento ${ }^{(32)}$.
Quanto aos problemas na implementação do plano de alta a mulheres submetidas a mastectomia, foi realizada uma pesquisa com 54 enfermeiros que trabalham em enfermarias sobre a compreensão, adesão e barreiras do planejamento de alta. Constatou-se que a adesão à política de planejamento de alta é baixa por parte dos enfermeiros, embora haja conscientização sobre a qualidade reduzida dos desfechos dos pacientes, em especial às submetidas à mastectomia. Nesse aspecto, as barreiras mais comuns ao planejamento de alta identificadas foram a falta de tempo e fatores relacionados à paciente mastectomizada(33).

Ademais, é interessante relatar que os atrasos desnecessários na alta de pacientes do hospital para casa são um problema constante e, para pessoas com mais idade, isso pode resultar em agravos para a sua saúde. Embora haja desafios na obtenção de excelentes práticas na transferência do atendimento de um paciente do hospital para o domicílio, há uma disposição e um compromisso dos enfermeiros no que diz respeito à comunidade e no hospital com a finalidade de melhorar a experiência da paciente ${ }^{(34)}$.

Em uma pesquisa ${ }^{(35)}$ desenvolvida com 16 enfermeiros de três hospitais diferentes na Suécia acerca dos desafios no cuidado às mulheres mastectomizadas evidenciou que cuidar desse tipo de paciente requer vários aspectos. Os enfermeiros relataram sentir demandas irrealistas ao cuidar delas, as quais são referentes a própria profissão e ao conhecimento sobre o cuidado com essas mulheres.

Em um trabalho ${ }^{(36)}$ feito com 27 enfermeiros em um hospital australiano de referência terciária metropolitana em 2011 sobre o planejamento de alta evidenciou que esta ferramenta é muito complexa e amplamente desvalorizada. Nesse caso, há diferenças significativas e mal compreendidas no 
que diz respeito à priorização e no atendimento de pacientes submetidas à mastectomia.

Então, é elucidado que uma das principais razões para as reinternações é a falta de preparo adequado do paciente e família para a alta hospitalar. Diante disso, é primordial que a assistência de enfermagem seja repensada de maneira organizada, multidisciplinar e que envolva esse preparo para reduzir as necessidades e expectativas dessas pacientes e seus familiares em relação ao cuidado a ser prestado no contexto domiciliar ${ }^{(37)}$.

Por fim, foi desenvolvido um estudo $^{(38)}$ sobre a ocorrência das reinternações hospitalares em mulheres submetidas à mastectomia no período de janeiro de 2008 a dezembro de 2012, e evidenciou que a região Sudeste é o local onde foi realizado um maior número de mastectomias (23 mil procedimentos) se comparada a outras regiões (Norte 8.443, no Sul 2.280, no Centro-Oeste e 10.538 no Noroeste. Nesse contexto, o autor relata que as intervenções de enfermagem são primordiais neste processo com o objeti- vo de reduzir a incidência de hospitalizações e possíveis complicações.

\section{CONCLUSÃO}

A maioria dos artigos evidenciou que as intervenções de enfermagem no plano de alta a mulheres submetidas à mastectomia foram baseadas na educação continuada dessas mulheres e também de seus familiares. Essa ferramenta consiste em orientações baseadas na utilização de drenos, apoio emocional, possibilidade de esvaziamento axilar, identificação de sinais de infecções e manejo nos curativos.

Nesse aspecto, a educação continuada também envolve uma abordagem integral, humanizada e individualizada das mulheres submetidas à este procedimento cirúrgico, uma vez que elas apresentam necessidades reais que variam conforme a cultura e estilo de vida. Ademais, o planejamento de alta deve ser feito antes da saída formal das mulheres do hospital com o objetivo de evitar o acúmulo de informações e falta de compreensão com relação às orientações prestadas.
Outras intervenções citadas nesta pesquisa foram: o uso de livros, guias on-line, folhetos informativos, orientações no período pré-operatório e grupos de apoio promovidos pelos enfermeiros. Essas ferramentas, se desenvolvidas de maneira adequada, podem ser muito úteis no plano de alta promovido pelos enfermeiros.

Com relação às barreias na implementação do plano de alta em mulheres submetidas à mastectomia, foram identificadas: desvalorização, falta de preparo da paciente e da família após a alta, demandas irrealistas sobre a própria profissão e ao conhecimento sobre o cuidado com essas mulheres, atrasos desnecessários na alta de pacientes do hospital para casa, a falta de tempo e fatores relacionados a pacientes submetidos a mastectomia.

Sendo assim, outros estudos deverão ser realizados com o intuito de solucionar falhas com relação aos obstáculos enfrentados pelos enfermeiros na implementação do plano de alta em mulheres submetidas à mastectomia, uma vez que isso pode contribuir para o aprimoramento do cuidado deste profissional. 1

\section{Referências}

1. Instituto Nacional do Câncer. Conceito e magnitude do controle do câncer de mama [Internet]. 2017 [acesso em 2018 jul 27]. Disponivel em: http://www2.inca.gov.br.

2. Soares PBM, et al. Características das mulheres com câncer de mama assistidas em serviços de referência do Norte de Minas Gerais. Rev Bras Epidemiol [Internet]. 2012 [acesso em 2018 jul 27]; 15(3): 595-604. Disponivel em: http://www.scielo.br/scielo.php?script=sci_ arttext\&pid=S1415-790X2012000300013.

3. Rodrigues, JD, Cruz, MS, Paixão, AN. Uma análise da prevenção do câncer de mama no Brasil. Ciênc. saúde coletiva [Internet]. 2015 out [acesso em 2018 jul 27]. 20(10). Disponível em: http://www.scielo.br/scielo. php?pid=S1413-81232015001003163\&script=sci_abstract\&tlng=pt.

4. Cavalcante SAM, et al. Ações do Enfermeiro no rastreamento e Diagnóstico do Câncer de Mama no Brasil. Revista Brasileira de Cancerologia. 2013; 59(3):456-66.

5. Mineo FLV, et al. A assistência de enfermagem no tratamento do câncer de mama. Revista Eletrônica Gestão \& Saúde [Internet]. 2013 [acesso em 2018 jul 27].4(2): 2238-2260. Disponível em: https://periodicos.unb.br/index.php/rgs/article/viewFile/22951/16474.

6. Tiezzi, DG. Epidemiologia do câncer de mama. Rev Bras Ginecol Obstet [Internet]. 2009 [acesso em 2018 jul 27].31(5): 213-5. Disponível em: http://www.scielo.br/pdf/rbgo/v31n5/v31n5a01.pdf.

7. Ohl ICB, et al. Ações públicas para o controle do câncer de mama no
Brasil: revisão integrativa. Rev Bras Enferm [Internet]. 2016 [acesso em 2018 jul 27]; 69(4): 746-55. Disponível em: http://www.scielo.br/ scielo.php?pid=S0034-71672016000400793\&script=sci_abstract\&tl$\mathrm{ng}=\mathrm{pt}$.

8. Silva SED, et al. Representações sociais de mulheres mastectomizadas e suas implicações para o autocuidado. Rev Bras Enferm [Internet]. 2010 [acesso em 2018 jul 27]; 63(5): 727-64. Disponivel em: http:// www.scielo.br/scielo.php?pid=S0034-71672010000500006\&script=sci_abstract\&tlng=pt.

9. Conselho Regional de Enfermagem de São Paulo. Parecer Coren-SP Cat 023/2010 [Internet]. 2010 [acesso em 2018 jul 27].Disponível em: http://portal.coren-sp.gov.br/sites/default/files/parecer_coren sp_2010_23.pdf.

10. Mendes KDS, Silveira RCCP, Galvão CM. Revisão integrativa: método de pesquisa para a incorporação de evidências na saúde e na enfermagem. Texto Contexto Enferm [Internet]. 2008 out-dez [acesso em 2018 jul 27]; 17(4): 758-64, 2008. Disponível em: http://www.scielo.br/scielo.php?script=sci_arttext\&pid=S0104-07072008000400018. 11. Santos CMC, Pimenta CAM, Nobre MRC. A estratégia PICO para a construção da pergunta de pesquisa e busca de evidências. São Paulo: Revista Latino-Am Enferm [Internet]. 2007 [acesso em 2018 jul 27]. Disponível em: http://www.scielo.br/scielo.php?pi$\mathrm{d}=$ S010411692007000300023\&script=sci_arttext\&tlng=pt. 


\section{Referências}

12. Lopes ALM, Fraciolli LA. Revisão Sistemática de Literatura e Metassíntese Qualitativa: considerações sobre sua aplicação na pesquisa em enfermagem. Texto e Contexto Enfermagem [Internet]. 2008 [acesso em 2018 jul 27]. Disponivel em: https://www.researchgate.net/publication/262760350_Systematic_review_of_literature_and_qualitative_metasynthesis_considerations_about_their_application_in_nursing_research.

13.Moher D, Libertati A, Tetzalaff J, Altman DG. The PRISMA Group. Group. Preferred Reporting Items for Systematic Reviews and Meta-Analyses: The PRISMA Statement [Internet]. [acesso em 2018 jul 27]. Disponivel em: www.prisma-statement.org.

14.Paiva ACPC, et al. Construção de instrumentos para o cuidado sistematizado da enfermagem: Mulheres em processo cirúrgico de mastec tomia. Rev. enferm. Cent.-Oeste Min [Internet]. 2016 [acesso em 2018 jul 27]. Disponível em: http://www.seer.ufsj.edu.br/index.php/recom/ article/view/707.

15. Soares SGSC, Albuquerque JOL. Intervenção do enfermeiro no tratamento quimioterápico de mulheres com câncer de mama. Revista Saúde me Foco [Internet]. 2014 jan/jun [acesso em 2018 jul 27]; 1(1): 1-16. Disponivel em: http://www4.fsanet.com.br/revista/index.php/ saudeemfoco/article/view/156/380.

16. Silva GNC, et al 0 cuidado de enfermagem vivenciado por mulheres mastectomizadas. Revista HU-UFJF [Internet]. 2013 [acesso em 2018 jul 27]; 39(1):1-10. Disponível em: https://hurevista.ufjf.emnuvens.com.br/ hurevista/article/view/1970.

17. Martins KP, et al. Atuação do enfermeiro no preparo para a alta hospitalar de pacientes cirúrgicos. Rev. pesqui. cuid. fundam. (Online) [Internet]. 2015 [acesso em 2018 jul 27]. DOI: 10.9789/2175-5361.2015. v7i1.1756-1764.

18. Razdan SN, et al. Quality of life among patients after bilateral prophylactic mastectomy: a systematic review of patient-reported outcomes. Qual Life Res [Internet]. 2016 [acesso em 2018 jul 27].Disponível em: https://www.ncbi.nlm.nih.gov/pubmed/26577764.

19. Toye C, et al. Outcomes for family carers of a nurse-delivered hospital discharge intervention for older people (the Further Enabling Care at Home Program): Single blind randomised controlled tria. International Journal of Nursing Studies [Internet]. 2016 [acesso em 2018 jul 27]. Disponivel em: https://www.ncbi.nlm.nih.gov/pubmed/27684320>8.

20. Wrobleski DM, et al. Discharge planning rounds to the bedside: a patient- and family-centered approach. Med surg nursing [Internet]. 2014 [acesso em 2018 jul 27]. Disponível em: https://www.ncbi.nlm. nih.gov/pubmed/24933789.

21. Bench $S$, et al. Effectiveness of critical care discharge information in supporting early recovery from critical ilness. Critical Care Nurse [Internet]. 2013 [acesso em 2018 jul 27]. Disponível em: https://www. ncbi.nlm.nih.gov/pubmed/23727851.

22. Nicolau SRTC, Teixeira ER. Clinical protocol for the nursing care of mastectomized women: an exploratory descriptive study. Revista Brasileira de Enfermagem Online [Internet]. 2014 [acesso em 2018 jul 27]. Disponível em: http://www.objnursing.uff.br/index.php/nursing/article/ view/4761/html_254.

23. Dal Molin A, et al. Hospital discharge: Results from an Italian multicenter prospective study using blaylock risk assessment screening score. International Journal of Nursing Knowledge [Internet]. 2014 [acesso em 2018 jul 27]. Disponível em: https://www.ncbi.nlm.nih.gov/ pubmed/24299656.

24. Bordallo FR, et al. The education as a facilitator practice of the process of care to mastectomized women: an integrative review. Revista de Enfermagem UFPE [Internet]. 2013 [acesso em 2018 jul 27]. Disponível em: https://periodicos.ufpe.br/revistas/revistaenfermagem/ article/view/12382.
25. Lambert L, Rusby J. Women's Viewl of 23-Hour Hospital Dischargel Following Mastectomy. Cancer Nursing Practice [Internet]. 2014 [acesso em 2018 jul 27]. Disponível em: https://journals.rcni.com/cancer-nursing-practice/womens-view-of-23hour-hospital-discharge-following-mastectomy-cnp2014.03.13.2.18.e1043.

26. Cho HSM, et al. A randomised trial of nursing interventions supporting recovery of the postmastectomy patient. Journal of Clinical Nursing [Internet]. 2013 [acesso em 2018 jul 27]. Disponivel em: https:// www.ncbi.nlm.nih.gov/pubmed/22830927.

27. Sakai S, et al. Effect of a Discharge Planning Educational Program in a University Hospital. Gan to kagaku ryoho. Cancer \& Chemotherapy [Internet]. 2015 [acesso em 2018 jul 27]. Disponivel em: https://www. ncbi.nlm.nih.gov/pubmed/26809418.

28. Kanekkaril G, et al. The Dismall Dischargel Process:a quality Improvement Project to enhance the timeliness and completion of discharge prescriptions. American Journal of Physical Medicine \& Rehabilitation [Internet]. 2014 [acesso em 2018 jul 27]. Disponivel em: https://www. ncbi.nlm.nih.gov/pmc/articles/PMC4652709/.

29. Nicolau SRTC. Cuidados sensiveis de enfermagem a mulheres submetidas a mastectomia [Dissertação]. 2015 [Internet]. Niterói: Universidade Federal Fluminense [acesso em 2018 jul 27]. Disponível em: https://app.uff.br/riuff/bitstream/1/3059/1/Sandra $\% 20$ Regina $\% 20$ Terra\%20Campos\%20Nicolau.pdf.

30. Rodrigues C. Qualidade de vida em mulheres adultas mastectomizadas [Dissertação]. 2017 [Internet]. Viseu: Instituto Polítécnico de Viseu [acesso em 2018 jul 27]. Disponível em: http://repositorio.ipv. pt/bitstream/10400.19/4749/1/ClaraMariaMoreiraGuedesRodrigues_ DM.pdf.

31. Carvalho APR, Santos TCB, Linhares FMP. Promoção do autocuidado a mulheres mastectomizadas. Cogitare Enferm [Internet]. 2012 jul/set. [acesso em 2018 jul 27]; 17(3):485-91. Disponivel em: https://revistas. ufpr.br/cogitare/article/view/29290.

32. Almeida NG, et al. Qualidade de vida e cuidado de enfermagem na percepção de mulheres mastectomizadas. Rev Enferm UFSM [Internet]. 2015 out./dez [acesso em 2018 jul 27]; 5(4):607-617. Disponível em: https://periodicos.ufsm.br/reufsm/article/view/17103/pdf.

33. Graham J, et al. Nurses' discharge planning and risk assessment: Behaviours, understanding and barriers. Journal of Clinical Nursing [Internet]. 2013 [acesso em 2018 jul 27]. Disponível em: https://www.ncbi. nlm.nih.gov/pubmed/23581501.

34. Pellet C. Discharge planning: Best practice in transitions of care. British Journal of Community Nursing [Internet]. 2016 [acesso em 2018 jul 27]. Disponível em: https://www.ncbi.nlm.nih.gov/pub$\mathrm{med} / 27809581$.

35. Kauppi W, Pross M, Olausson S. Ward nurses' experiences of the discharge process between intensive care unit and general ward Nursing in Critical Care [Internet]. 2018 [acesso em 2018 jul 27]. Disponivel em: https://www.ncbi.nlm.nih.gov/pubmed/29359426.

36. Cognet $S$, Coyer F. Discharge practices for the intensive care patient: A qualitative exploration in the general ward setting. Intensive \& Critical Care Nursing [Internet]. 2014 [acesso em 2018 jul 27]. Disponível em: https://www.ncbi.nlm.nih.gov/pubmed/24907890.

37. Souza MB, Quelucci CG. Consideraions on Nursing Care to patients ai Hospital Discharge: integrative review. Crise [Internet]. 2013 [acesso em 2018 jul 27]. Disponível em: https://www.ncbi.nlm.nih.gov/pub$\mathrm{med} / 27809591$

38. Valle PM, et al. Mastectomy Due to Breast Cancer in Brazil: Geographic Distribution and Costs from the Public Health Care Perspective. Value in Health [Internet]. 2014 [acesso em 2018 jul 27]. Disponível em: https://www.valueinhealthjournal.com/article/S1098-3015(14)041758/fulltext. 